\title{
Patient Perspectives on Delivery of Drug Therapy Information and Renal Pharmacist Quality Indicator - Drug Therapy Problems (QI-DTPs)
}

Mary T Lam BSc. M(Pharm), Natalie Lesko BSc(Pharm), ACPR, PharmD, William Nevers BSc(Pharm), ACPR, PharmD, Sean Gorman BSc(Pharm), ACPR, PharmD, Kate Boutin BSc. BSc(Pharm), ACPR, Alice Ratcheva BSc(Pharm), ACPR

\section{Background}

Chronic kidney disease (CKD) patients have complex

medication regimes $=$ high risk of DTPs

Consensus of renal pharmacist 17 QI-DTPs developed in 2015/16 to improve renal pharmacy practice \& patient care

Barriers and facilitators for renal pharmacist uptake of QI-DTPs in practice identified in 2016/17

- Patient and family centered care identified as a priority by the Ministry of Health and Interior Health Authority

\section{Objectives}

To determine the type of information renal patients require to make decisions about drug therapy

To determine the type of medication-related information renal patients would like to enable them to adhere to their medication regimen

To obtain patient input on a previously developed list of renal pharmacist QI-DTPs

To help inform the development of an intervention to increase the uptake of renal pharmacist QI-DTPS

Methods

Design

- Prospective, single center, qualitative study

Setting and sampling

- Kelowna General Hospital Renal Clinic

- Purposeful sample

Inclusion

- CKD patients: Stage 3, 4 and 5

- $\geq 1$ of Diabetes, Hypertension or Cardiovascular Disease Data collection

Semi-structured, 1-on-1 in-person or telephone interviews using interview guide developed by study investigators

Interviews transcribed by one study investigator

Data analysis

Transcript-based thematic analysis

- Consensus of coding and themes for each interview
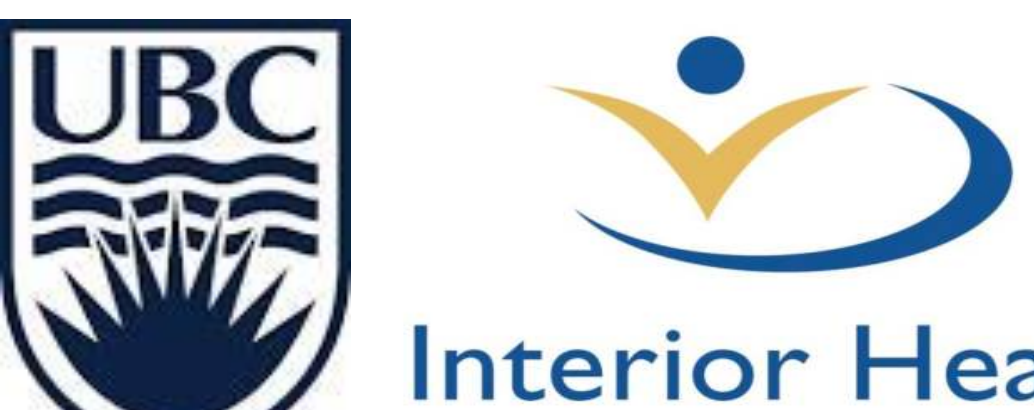

Interior Health

\section{Results}

\begin{tabular}{|c|c|}
\hline \multicolumn{2}{|c|}{ Table 1. Demographics } \\
\hline Characteristic & $\mathbf{n}=10$ \\
\hline Age (years) & 70 \\
\hline Female & $5(50 \%)$ \\
\hline Diabetes & $6(60 \%)$ \\
\hline Hypertension & $9(90 \%)$ \\
\hline Cardiovascular Disease & $5(50 \%)$ \\
\hline CKD Etiology & \\
Hypertension & $1(10 \%)$ \\
Diabetes & $2(20 \%)$ \\
Multi-factorial & $6(60 \%)$ \\
\hline CKD Stage & \\
3 & $5(50 \%)$ \\
4 & $2(30 \%)$ \\
5 & $20 \%)$ \\
\hline
\end{tabular}

\section{Table 3. Information to Support} Adherence

Barriers to Medication Adherence

\begin{tabular}{l|c|} 
Code & Utterances \\
(Participants)
\end{tabular}

Enablers to Medication Adherence

Code

Utterances

\begin{tabular}{c|c} 
& (Participants) \\
\hline Knowing benefits & $1(1)$
\end{tabular}

Additional information or discussion of benefits would not be helpful

$$
\mathrm{n}=9 \text { \#utt. } 21
$$

"I don't miss medications because I don't have the information about how it work to take them completely"

\begin{tabular}{|c|c|}
\hline \multicolumn{2}{|c|}{$\begin{array}{l}\text { Table 2. Decision-Making } \\
\text { Information \& Sources }\end{array}$} \\
\hline Helpful & $\begin{array}{c}\text { Utterances } \\
\text { (Participants) }\end{array}$ \\
\hline $\begin{array}{l}\text { Adverse Drug } \\
\text { Reactions }\end{array}$ & $16(9)$ \\
\hline Expected benefit(s) & $14(7)$ \\
\hline $\begin{array}{l}\text { Administration of } \\
\text { medication }\end{array}$ & $9(8)$ \\
\hline Medication indication & $8(7)$ \\
\hline \multicolumn{2}{|l|}{ Unhelpful } \\
\hline Confusing terms & $8(4)$ \\
\hline High volume & $4(2)$ \\
\hline \multicolumn{2}{|c|}{ Sources - Verbal } \\
\hline Physician & $52(9)$ \\
\hline Pharmacist & $46(10)$ \\
\hline \multicolumn{2}{|c|}{ Sources - Written } \\
\hline Pharmacy Handout & $17(9)$ \\
\hline Internet-Based & $10(6)$ \\
\hline
\end{tabular}

Table 4a. Input on QI-DTPs

Expectation of medications

\begin{tabular}{|c|c|}
\hline Code & $\begin{array}{c}\text { Utterances } \\
\text { (Participants) }\end{array}$ \\
\hline
\end{tabular}

Slow progression $8(8)$

of CKD

Better health /

$8(8)$

Improve health

$5(5)$

General priorities or concerns

\begin{tabular}{|c|c|} 
Code & $\begin{array}{c}\text { Utterances } \\
\text { (Participants) }\end{array}$ \\
\hline $\begin{array}{c}\text { Decrease \# of } \\
\text { medications }\end{array}$ & $8(8)$ \\
\hline $\begin{array}{c}\text { Optimize } \\
\text { medication therapy }\end{array}$ & $3(3)$ \\
\hline
\end{tabular}

\begin{tabular}{|c|c|c|}
\hline \multicolumn{3}{|l|}{ Results } \\
\hline \multicolumn{3}{|c|}{$\begin{array}{c}\text { Table } 4 \mathrm{~b} . \\
\text { Input on QI-DTPs }\end{array}$} \\
\hline \multicolumn{3}{|c|}{$\begin{array}{c}\text { Example - Diabetes QI-DTP } \\
\text { In patients with above normal A1c, add medications to } \\
\text { decrease A1c to prevent further damage to the kidneys }\end{array}$} \\
\hline High Priority & Code & $\begin{array}{l}\text { Utterances } \\
\text { (Participants) }\end{array}$ \\
\hline \multirow[t]{2}{*}{$\begin{array}{l}\text { Diabetes } \\
n=4(6)\end{array}$} & $\begin{array}{c}\text { Diabetes can damage my } \\
\text { kidneys }\end{array}$ & $2(2)$ \\
\hline & $\begin{array}{l}\text { Important to optimize } \\
\text { blood glucose }\end{array}$ & $1(1)$ \\
\hline \multirow[t]{2}{*}{$\begin{array}{l}\text { Hypertension } \\
\qquad \mathrm{n}=8 \text { (9) }\end{array}$} & $\begin{array}{l}\text { Slow progression of } \\
\text { kidney disease }\end{array}$ & $4(4)$ \\
\hline & Takes an ACE-inhibitor & $2(2)$ \\
\hline \multirow{2}{*}{$\begin{array}{c}\text { Prevention of CVD } \\
n=5(5)\end{array}$} & Previous adverse event & 2(3) \\
\hline & Prevent CV event & 1(1) \\
\hline \multicolumn{3}{|l|}{ Limitations } \\
\hline \multicolumn{3}{|c|}{$\begin{array}{l}\text { - } 1 \text { in-person interview, } 9 \text { over the telephone } \\
\text { - } \text { Could not code portions of one interview } \\
\text { Study timeline and recruitment limited number of } \\
\text { participants }\end{array}$} \\
\hline \multicolumn{3}{|l|}{ Conclusions } \\
\hline \multicolumn{3}{|c|}{$\begin{array}{l}\text { CKD Patients: } \\
\text { - Want to be involved in medication decisions } \\
\text { Information: } \\
\text { - Patients are interested in indication, mechanism of action, } \\
\text { expected benefits/risks and how to use their medication } \\
\text { - Medical terminology can be confusing } \\
\text { - } \text { Find high volume of written information unhelpful } \\
\text { help with adherence }\end{array}$} \\
\hline \multicolumn{3}{|c|}{$\begin{array}{l}\text { QI-DTPs: } \\
\text { - High priority to patients based on their current medications } \\
\text { improve health }\end{array}$} \\
\hline
\end{tabular}

